\title{
A Truthful Auction for Access Point Selection in Heterogeneous Mobile Networks
}

\author{
Stefano Paris*, Fabio Martignon ${ }^{\dagger}$, Ilario Filippini ${ }^{\ddagger}$ and Antonio Capone ${ }^{\ddagger}$ \\ ${ }^{*}$ Dep. of Information Technology ${ }^{\dagger}$ Lab. de Recherche en Informatique ${ }^{\ddagger}$ Dip. di Elettronica e Informazione \\ and Math. Methods \\ Université Paris-Sud 11 \\ University of Bergamo \\ fabio.martignon@1ri.fr \\ Politecnico di Milano
}

stefano.paris@unibg.it

\begin{abstract}
In recent years, with the evolution of new and content-rich Internet services, mobile network operators face the challenging task to guarantee ubiquitous access to their customers, while minimizing network deployment costs.

In order to foster the opportunistic utilization of unexploited Internet connections of residential users, we propose a new marketplace where mobile network operators can rent the unused capacity of residential users' access devices (e.g., wireless access points or femtocells) when the traffic demand of their mobile customers exceeds the operator's network capacity. We formulate the allocation problem as a combinatorial reverse auction, which prevents market manipulation, and we further propose a greedy algorithm that finds efficient allocations in polynomial time, even for large-size network scenarios.

Numerical results demonstrate that our proposed schemes well capture the economical and networking essence of the allocation problem, thus representing a promising approach to enhance the performance of next-generation wireless access networks.
\end{abstract}

Index Terms-Community Networks, Heterogeneous Wireless Access Networks, Pricing Policy.

\section{INTRODUCTION}

In recent years, the rapid growth of the bandwidth demand required by content-rich Internet services accessed by mobile users through their 3G/3GPP devices has increased the pressure on mobile network operators for the upgrading of their cellular networks. Nevertheless, the increasing installation and management costs might limit the deployment of new base stations to provide the bandwidth required for accessing Internet services. This problem might further worsen with the integration of energy efficient mechanisms [1], which switch off the underused base stations in order to reduce the power consumption, since, as a side effect, they also reduce the overall bandwidth of the mobile network.

Several works investigate the benefit of opportunistically exploiting WiFi access networks to improve the QoS experienced by $3 \mathrm{G}$ devices [2], [3], [4]. Consequently, mobile network operators could provide a better service without limiting the maximum traffic of their customers through a wise management of their resources and the opportunistic utilization of other access networks.

In this paper, we therefore propose a new marketplace which acts as an incentive for exploiting opportunistically the unused Internet connections of residential users. In particular, we envision a marketplace scenario for the unexploited bandwidth of the devices used by residential users for Internet access, which can be shared or even sold to third party devices through a wireless connection, like in Wireless Community Networks [5]. A mobile network operator rents the capacity made available by residential users through their access devices in order to satisfy the additional traffic demand that mobile customers need for accessing content-rich web services.

As any marketplace, the misbehavior of even few residential users playing strategically might seriously affect the efficiency of the allocation mechanism used by the mobile network operator, thus discouraging the operator and residential users from participating to the market. To address this issue, we present a reverse truthful auction targeted for the heterogeneous network scenario described above, which forces each residential user interested in leasing the unexploited bandwidth of its Internet connection to ask its real valuation for such capacity.

The temporary agreements that can be stipulated by the mobile network operator and residential users through our marketplace are economically efficient, since the operator can provide a better service to its mobile customers without increasing the installation costs of its network, while residential users can earn money from their unexploited Internet connection.

In an effort to design a mechanism which would boost economically the deployment of heterogeneous networks, our work makes the following contributions:

- We propose and analyze an innovative marketplace for selecting the cheapest available Access Points to serve the additional traffic demand of mobile users.

- We propose a combinatorial truthful auction that minimizes the leasing costs sustained by a mobile network operator, which is resilient against any market manipulation.

- Since the optimal reverse auction problem is NP-Hard, we further propose a greedy algorithm that solves very efficiently (i.e., in polynomial time) the allocation problem, also for large network instances, while preserving the truthfulness property.

- We perform a numerical analysis and comparison of the proposed optimal and greedy allocation algorithms, considering several real-size network scenarios. 
The rest of this paper is structured as follows: Section II discusses related work. Section III presents the network and economic models considered in our work. Section IV formulates the combinatorial auction as an optimization model, while Section V describes the greedy algorithm to solve efficiently the problem. Finally, conclusions are discussed in Section VII.

\section{RELATED WORK}

In recent years, auction theory has turned out to be a powerful tool to solve efficiently resource allocation problems in several networking contexts.

In particular, with the upcoming generation of cognitive radio networks, market-based auctions have been extensively studied as an efficient mechanism to dynamically sublease the unexploited licensed spectrum to secondary users and increase the revenue of the spectrum owner [6], [7], [8], [9], [10].

VERITAS [6] pinpoints the limits of conventional auctions and proposes a truthful and flexible mechanism requiring only polynomial complexity for solving the spectrum allocation problem. TRUST [7] further develops this approach to support multi-party spectrum trading through a truthful double spectrum auction based on the well-known McAfee mechanism. The work presented in [8] adopts a similar approach to model and solve a broad class of problems concerning the allocation of spectrum resources to primary and secondary users in cognitive radio networks, while [9] analyzes also the interplay among the spectrum broker, service providers which are interested in leasing spectrum bands, and end-users. The analysis determines the conditions for the pricing policy that allow both service providers and end users to maximize their utilities. In [10] the authors investigate a spectrum marketplace where the spectrum owner's uncertainty about the private valuations of spectrum bidders is modeled using a Bayesian approach. Under this model, the authors propose an efficient algorithm to maximize the spectrum owner's expected revenue, while exploiting efficiently the spectrum resources.

Auction theory has also been exploited to design innovative traffic engineering techniques and routing protocols, both to enhance the utilization of unused network paths and force the collaboration of intermediate relaying nodes [11], [12], [13], [14], [15].

Finally, recent research has analyzed virtual network scenarios where several service providers compete among each other for using the resources owned and managed by a network operator [16], [17]. In particular, Jain et al. in [16] present a mechanism for per-link bandwidth allocation of end-to-end paths in wired network, whereas Fu et al. in [17] design an auction-based stochastic game for resource allocation of virtual operators in wireless cellular networks.

Unlike recent literature, our work envisions a new marketplace based on reverse auctions, where access devices of community networks (either WiFi Access Points or Femtocells) are exploited by mobile network operators to serve their customers. This marketplace would reduce the installation and management costs for mobile network operators, as well as foster the development of community networks.

\section{System ModeL}

This section presents the communication and network models considered in our work, as well as the definitions and assumptions we adopt in the design of our auction mechanisms.

Let us refer to the Heterogeneous Network (HN) scenario illustrated in Figure 1, which is composed of a Mobile Network (MN) formed by three Base Stations and a set of wireless access devices (APs) connected to the Internet (like, for example, Wifi access points or cellular femtocells).

The $\mathrm{MN}$ is managed by a single operator that provides ubiquitous access to its mobile customers (MCs), while each residential user, which participates to the marketplace, is the owner of a wireless access device (AP).

Residential users lease the unused capacity of their wireless access devices, so that the mobile operator can rent the available APs' bandwidth when the traffic demand of its customers exceeds the capacity provided by its mobile network.

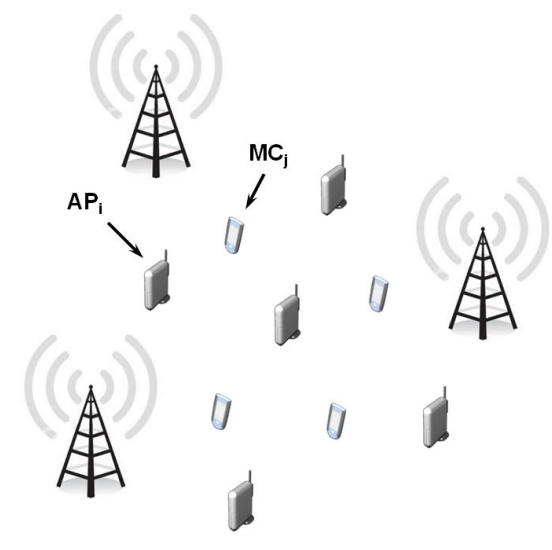

Fig. 1: Network scenario considered in this work. The MN is managed by a single operator that provides access to its customers (e.g., $M C_{j}$ ), while residential users lease the unused capacity of their wireless access devices (e.g., $A P_{i}$ ).

The mechanism we propose selects the residential access devices that are used to satisfy the MCs traffic demand which exceeds the MN capacity. More specifically, the MN operator leases the available bandwidth provided by the access devices, which in turn is used to satisfy its mobile customers.

Each seller ${ }^{1} i$ owns an AP that he is willing to lease for a given price $a_{i}$. The MN operator decides which access points (APs) are selected to satisfy the extra-demand of its mobile customers (MCs), and the price paid to the winners, $p_{i}$, for exploiting their devices.

We remark that, unlike classical optimization approaches, where the operator knows exactly the cost of each device that it can install, in our scenario such information is hidden. To prevent market distortion, the mechanisms we propose in this paper force residential users to provide the true information about the valuation of their APs.

\footnotetext{
${ }^{1}$ In this paper we use interchangeably the terms sellers, residential users and access points, while we use the term buyer to refer to the mobile network operator.
} 
We further assume that APs use orthogonal channels; therefore, the different subsets of MCs assigned to each AP do not interfere with each other.

We observe that the transmission rate and the channel utilization required to satisfy the extra demand depend on the distance between the mobile customer device and the access point to which it is connected; hence, the allocation mechanism influences the number of access points that the MN operator uses to satisfy the extra traffic demand.

Therefore, the aim of the operator is to minimize its cost by allocating the traffic demand of its MCs to the cheapest APs, i.e., to those residential users that are willing to ask the lowest price for the utilization of their devices.

To this end, we design a truthful auction that, in addition to minimizing the overall price paid by the $\mathrm{MN}$ operator, prevents market distortion by forcing every residential user to declare and ask its true valuation, $v_{i}=a_{i}$.

Each residential user $i$ submits its bid, $a_{i}$, representing the price that the seller $i$ asks for leasing its AP to the MN operator. To simplify the discussion, in this paper we consider only one type of residential users. However, our model can be extended straightforwardly by considering different types of residential users (classified, for example, according to their hidden cost, wired connection, or residual bandwidth).

The MN operator turns the extra traffic demand of its mobile customers $d_{j}$ into a vector of channel utilizations, $\overrightarrow{o_{j}}=\left[\begin{array}{llllll}o_{j 1} & o_{j 2} & \ldots & o_{j i} & \ldots & o_{j n}\end{array}\right]$, where each pair $(j, i)$ refers to a possible allocation of MC $j$ to $\mathrm{AP} i$, whereas $n$ represents the number of APs in the network. Channel utilizations are computed as follows:

$$
o_{j i}=\frac{d_{j}}{r_{j i}^{(\max )}},
$$

where the element $o_{j i}$ represents the channel utilization perceived by AP $i$ when it satisfies the demand of MC $j$, and it is computed as the ratio between the required traffic demand $d_{j}$ and the maximum achievable transmission rate of the wireless link that might connect $\mathrm{MC} j$ and $\mathrm{AP} i, r_{j i}^{(\max )}$. Note that this latter value can be easily obtained from the MAC layer through a scanning of the wireless channels, which is performed periodically by all network devices.

The allocation problem that minimizes the overall cost sustained by the MN operator to use the APs of residential users can be solved designing a combinatorial single-minded reverse auction. Specifically, the auction run by the MN operator aims at finding the cheapest APs to serve the traffic demand of the mobile customers. Each seller is interested in handling at least one mobile customer (i.e., a set composed of a mobile customer or any superset of it), since, in this case, he is paid by the MN operator which, in turn, uses its AP to serve the mobile customers. Therefore, the private valuation of residential user $i$ can be defined according to Equation (2):

$$
\begin{aligned}
v_{i}\left(S_{i}\right) & = \begin{cases}v_{i}<0 & \text { if }\left|S_{i}\right| \geq 1 \\
0 & \text { otherwise }\end{cases} \\
S_{i} & =\{j \in \mathcal{M}: j \text { is assigned to } i\}
\end{aligned}
$$

where $\mathcal{M}$ represents the set of mobile customer devices (MCs). Note that both valuations $v_{i}$ and payments are negative, since the mechanism pays the players (i.e., the residential users) that participate to the marketplace.

We observe that MCs do not pay any additional price for the services provided through the residential APs (e.g., Internet access). Indeed, the MN operator benefits from using a subset of these APs, since it can satisfy the growing bandwidth demand of its customers without installing new Base Stations, which have high installation and operational costs.

Let us denote by $p_{i} \leq 0$ the price paid to residential user $i$ when its AP is used by the operator. Then, the utility of user $i$ is defined as the difference between its private valuation $v_{i}$ and the paid price paid to exploit the bandwidth of its AP, $p_{i}$, according to the following expression:

$$
u_{i}= \begin{cases}v_{i}-p_{i} & \text { if }\left|S_{i}\right| \geq 1 \\ 0 & \text { otherwise }\end{cases}
$$

Obviously, when the residential user's AP $i$ is not used, its utility is null, since both the paid price and its valuation are null.

\section{Optimal Reverse Auction for AP Selection}

This section presents the combinatorial auction mechanism we propose to select the wireless access points for satisfying the extra-demand of mobile customers.

We formalize the optimal and truthful auction mechanism in two steps. First, we present an Integer Linear Programming (ILP) model which provides the optimal allocation for the auction. Then, we describe the algorithm that, exploiting the allocation of the ILP model, makes the auction truthful. This algorithm computes the price paid to the residential users in such a way that the optimal strategy for each residential user $i$ is to ask its real valuation $v_{i}$ for the utilization of its available capacity $c_{i}$.

We first describe the meaning of the variables used in our model, then we provide the ILP description of the problem.

Let $\mathcal{M}$ denote the set of mobile customer devices (MCs), and $\mathcal{A}$ the set of wireless access points (APs) whose owners (the residential users) participate to the reverse auction of the mobile operator. Let us define $\mathcal{M}_{i} \subseteq \mathcal{M}, i \in \mathcal{A}$ as the set of MCs that are covered by AP $i$ (i.e., the MCs that are in the radio range of AP $i$ ).

We can now introduce the decision variables used in our ILP model. Binary variables $x_{i}, i \in \mathcal{A}$, indicate which residential users win the auction, i.e., the APs whose available capacity is exploited by the mobile operator to serve the extra-traffic of its MCs $\left(x_{i}=1\right.$ if the available capacity of AP $i$ is used, 0 otherwise).

Binary variables $y_{j i}, i \in \mathcal{A}, j \in \mathcal{M}$, provide the assignment of MCs to APs $\left(y_{j i}=1\right.$ if $\mathrm{MC} j$ is assigned to $\mathrm{AP} i, 0$ otherwise).

Given the above definitions and notation, the reverse com- 
binatorial auction problem can be stated as follows:

$$
\begin{array}{ll}
\max \sum_{i \in \mathcal{A}} a_{i} \cdot x_{i} & \\
\text { s.t. } & \forall i \in \mathcal{A}, \forall j \in \mathcal{M}_{i} \\
y_{j i} \leq x_{i} & \forall j \in \mathcal{M} \\
\sum_{i \in \mathcal{A}} y_{j i}=1 & \forall i \in \mathcal{A} \\
\sum_{j \in \mathcal{M}_{i}} y_{j i} o_{j i} \leq 1 & \forall i \in \mathcal{A} \\
\sum_{j \in \mathcal{M}_{i}} y_{j i} d_{j} \leq x_{i} c_{i} & \forall i \in \mathcal{A}, \forall j \notin \mathcal{M}_{i} \\
y_{j i}=0 & \forall i \in \mathcal{A}, \forall j \in \mathcal{M} .
\end{array}
$$

The objective function (4) maximizes the social welfare, which coincides with the expected cost sustained by the MN operator to exploit the APs provided by the residential users (recall that the value $a_{i}$ is negative, since user $i$ gets money from the mechanism).

Constraints (5) are coherence constraints ensuring that only the access points that win the auction can be used to serve mobile customer devices.

The set of constraints (6) provide full coverage of all those mobile customer devices that are served by the MN operator through the leased residential access points.

Constraints (7) and (8) prevent the allocation of an overall traffic demand that cannot be satisfied by an access point, due to the maximum achievable transmission rate of the wireless channel and the limited capacity of the Internet connection made available by the residential user, while constraints (9) avoid the assignment of MCs to APs that are not in the reciprocal radio range. Note that the channel assignment of access points can be optimized in order to reduce interference effects among nearby devices.

Finally, constraints (10) ensure the integrality of the binary decision variables.

Having defined the ILP model representing the optimal auction, we now illustrate the algorithm that forces residential users to ask their real valuation for the utilization of the capacity that they make available through their access points.

Algorithm 1 describes the steps performed by the MN operator to select the cheapest APs. The algorithm receives as input the parameters which describe the network topology and all residential users' offers; these latter are composed of the capacity $c_{i}$ made available through the APs and its cost $a_{i}$. It produces as output the allocation of mobile customer devices

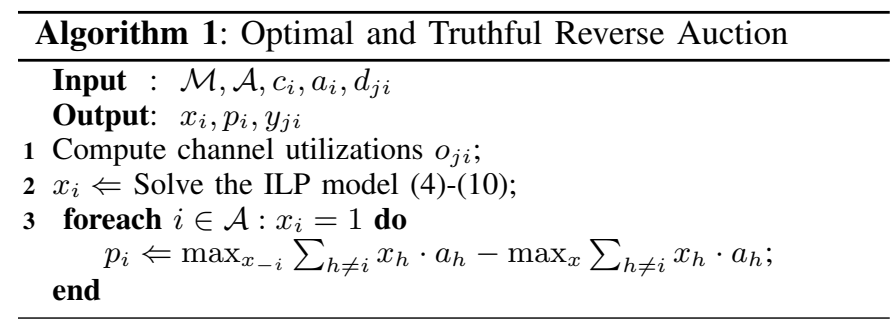

to access points, $y_{j i}$, as well as the price $p_{i}$ paid to each winning residential user to exploit the available capacity of its access point $\left(\exists p_{i} \leq 0, \forall i \in \mathcal{A}: x_{i}=1\right)$.

The algorithm proceeds in 3 steps. In step 1, the traffic demands of mobile customers are transformed into equivalent channel utilizations, using both their extra traffic demand and the achievable transmission rate of the links that can be established with all nearby APs, according to Equation (1). Step 2 consists in solving the ILP model to find the allocation that maximizes the social welfare, or equivalently, the expected cost paid by the operator. Finally, in step 3, the operator computes the prices paid to the winners according to the Grove rule, which guarantees a truthful auction.

\section{Greedy Reverse Auction for AP Selection}

The optimal auction problem described in the previous section is NP-Hard, and the computation time necessary to solve large network instances increases very sharply. Note, however, that the truthfulness property guaranteed by the payment scheme proposed for Algorithm 1 is no longer satisfied if the combinatorial auction is not solved to the optimality, but only approximated [18].

For the reasons mentioned above, in the following we present a greedy algorithm to solve efficiently (i.e., in polynomial time) the allocation problem, preserving the truthfulness property, so that revealing the true valuation is the dominant strategy for all residential users who participate to the approximated bandwidth allocation auction.

The greedy auction is summarized in Algorithm 2, and it is composed of two main phases: (1) the allocation phase, which selects the APs in descending order of their bids per number of mobile customers which they may cover (recall that $a_{i} /\left|\mathcal{M}_{i}\right|<0$ ), until the whole traffic demand generated by mobile customers is satisfied, and (2) the payment phase, which establishes the price paid to each winner as a function of the first unused AP in the sorted list (the first looser). This latter is also referred to as critical access point for $i$ and the price asked by its owner as critical value for $i$, which will be denoted as $a_{c}$.

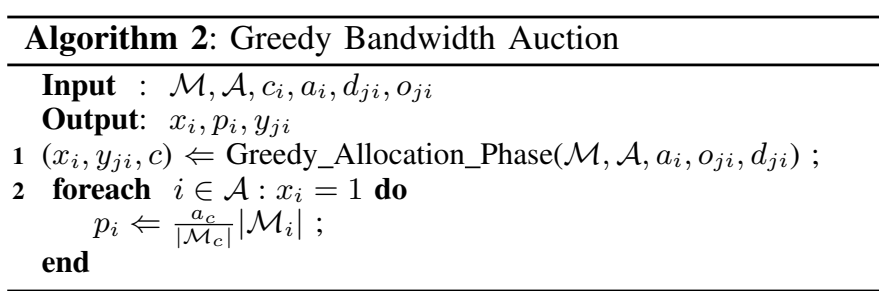

The greedy allocation phase, which is detailed in Algorithm 3, sorts the set of APs in non increasing order of their submitted bids per number of MCs which they may serve, $\frac{a_{i}}{\left|\mathcal{M}_{i}\right|}$. Then, each element of the sorted list is selected until all MCs are assigned to an AP. The assignment procedure assigns to any $\mathrm{AP} i \in \mathcal{A}$ selected as winner the maximum number of unsatisfied MCs in its radio range $\left(j \in \mathcal{M}_{i}: \sum_{h \in \mathcal{A}} y_{j h}=0\right)$ such that either the wireless channel is not saturated (i.e., its 


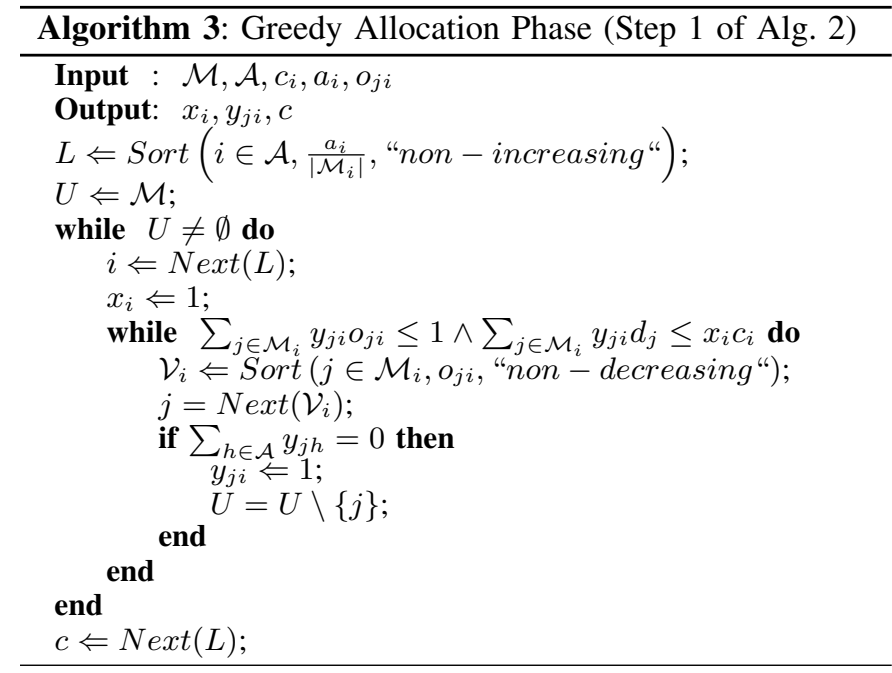

utilization is lower than 1) or the overall traffic demand does not exceed the capacity of the wired connection.

In addition to selecting the APs used by the operator and provide the allocation of the mobile customers to such APs, Algorithm 3 computes also the critical access point, which is the first unselected AP (or the last selected AP) of the sorted list.

We observe that Algorithm 2 implements a truthful auction. In fact, the allocation phase satisfies the monotonicity property (recall that the APs are sorted in non-increasing order of their bid per number of covered mobile customers), and there exists a critical value which determines if the residential user's AP is satisfied or not. Therefore, a MN operator can efficiently compute a solution for the reverse auction problem, being assured that all residential users reveal the true valuation for their AP available capacity.

\section{NUMERICAL RESULTS}

In this section, we illustrate the numerical results obtained solving the reverse auction with the optimal and greedy algorithms detailed in previous sections. We first describe the experimental methodology employed in our simulations, and then we measure the performance of our proposed solutions.

Experimental Methodology. In our simulations, we consider network topologies composed of 10 Access Points and 100 Mobile Customers randomly scattered over an area of $1000 \times 1000 m^{2}$. Both the extra traffic demands of MCs and the bids offered by residential users for their APs are drawn from uniform distributions; in particular, we vary the average values of both the MCs traffic demands and APs bids in the range $[0.5,2]$ Mbps and [10,20] monetary units (e.g., US dollars), respectively.

The channel capacity of both access and backbone links is defined according to the reception sensitivity of the Wistron CM9 commercial wireless cards (based on Atheros chipset) ${ }^{2}$. The path loss, which is necessary to evaluate the sensitivity

\footnotetext{
${ }^{2}$ Available on-line http://www.diswire.com/SpecsCM9.pdf
}

of the receiving node, is computed according to the Friis propagation model. We observe that all the above assumptions do not affect the proposed algorithms, which can be used to solve any network scenario.

In order to analyze and compare the performance of the proposed greedy algorithm (Section V) with the optimal solution (Section IV), we consider the following metrics:

- Total Cost: defined as the sum of the prices paid by the operator to all winners, $-\sum_{i \in \mathcal{A}} p_{i} \cdot x_{i}$.

- Social Welfare: defined as the sum of the winner bids, $-\sum_{i \in \mathcal{A}} a_{i} \cdot x_{i}$. This is the function maximized by our mechanisms, and represents the ideal bound for the Total Cost paid by the MN operator.

- Winners: defined as the number of winners selected among the APs that participate to the auction.

For each network scenario, we performed 10 independent measurements, computing very narrow $95 \%$ confidence intervals.

Performance Evaluation. Figure 2 shows the Total Cost and Social Welfare paid by the MN operator for three different mean values of the residential user bids measured in the network topologies composed of 10 access points and 100 mobile customers as a function of the average bandwidth demand of the customers traffic. The curves identified by labels "Opt." and "Greedy" illustrate the cost computed using the Optimal and Greedy algorithms, respectively, while the curve identified by "SW" corresponds to the Social Welfare, which represents the cost paid by the operator without implementing a payment rule that guarantees the truthfulness property. Indeed, this value represents an ideal lower bound to the Total Cost, since it can be achieved only assuming that all residential users behave honestly.

The difference between the Total Cost and the Social Welfare represents, therefore, the hidden cost paid by the operator to force residential users to submit the real valuation for the use of their APs.

We can observe that the overall cost paid by the operator using Algorithm 1 (the "Opt." curve) well approaches the Social Welfare in all network scenarios, as illustrated in Figure 2. Nevertheless, the high computation time necessary to solve the auction might discourage $\mathrm{MN}$ operators to use residential APs to serve their customers in the presence of high mobility. On the contrary, Algorithm 2 (the "Greedy" curve) finds a solution in polynomial time, thus representing an efficient alternative to solve the AP reverse auction. Note, however, that the higher price paid using the Greedy Algorithm is surely lower than the cost that would be paid to upgrade the capacity of the mobile network through the installation of new Base Stations. Furthermore, it can be observed that data traffic with low bandwidth demands increases the competition among residential users, resulting in a lower price paid by the operator. Therefore, a MN operator should determine the best trade-off between the average traffic demand and the cost that he is willing to pay to satisfy its customers, before performing the reverse auction. The definition of an algorithm to find such trade-off is left as a future issue. 


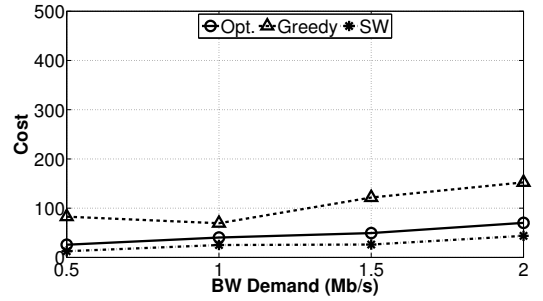

(a) $\mathrm{Bid}=10$

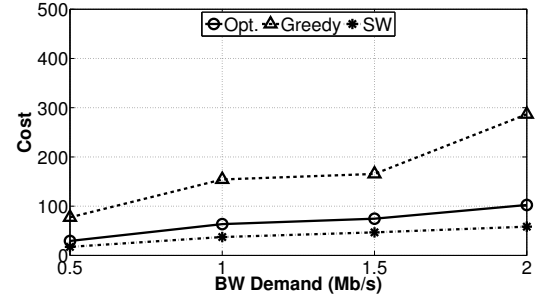

(b) Bid $=15$

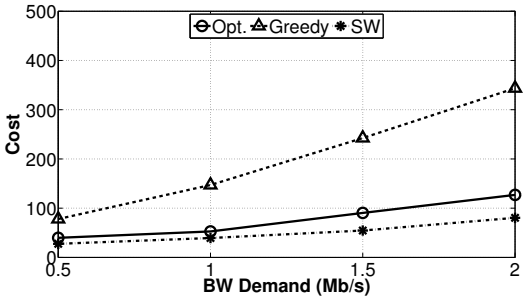

(c) Bid $=20$

Fig. 2: Costs and Social Welfare paid by the operator, measured as a function of the average traffic demand of mobile customers.

Figure 3 shows the fraction of APs selected as winners by Algorithms 1 and 2 as a function of the average bandwidth demand of the traffic generated by mobile customers. As expected, the higher is the average bandwidth of the customers traffic, the higher is the number of APs that the operator needs to satisfy its customers.

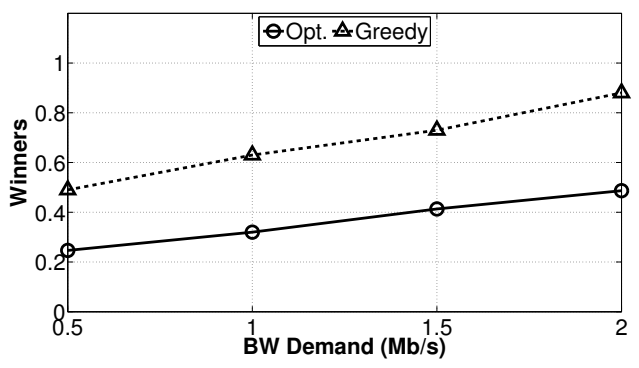

Fig. 3: Fraction of APs selected as winners by the Optimal and Greedy algorithms as a function of the average traffic demand of MCs.

\section{CONCLUSION}

This paper proposed a new marketplace where mobile network operators can rent the available bandwidth of Internet connections sold by residential users through their wireless access devices (either WiFi Access Points or femtocells).

The allocation problem faced by the mobile network operator is formulated as a combinatorial auction in order to minimize the cost payed for exploiting the residential user devices and prevent market manipulation (since this latter may decrease the effectiveness of the proposed mechanism), forcing all residential users to reveal their real valuation.

We proposed both an optimal, combinatorial truthful auction that minimizes the leasing costs sustained by the mobile network operator, which is resilient against any market manipulation, as well as a greedy algorithm that solves very efficiently (i.e., in polynomial time) the allocation problem also for large network instances, while preserving the truthfulness property.

Numerical results demonstrate that our proposed allocation schemes well capture the economical and networking essence of the problem, thus representing a promising solution to enhance the performance of next-generation wireless access networks.

\section{ACKNOWLEDGMENT}

This work was partially funded by the Italian PRIN 2009 project GATECOM (GATEway-based architecture for contentcentric COMmunity networking).

\section{REFERENCES}

[1] M.A. Marsan, L. Chiaraviglio, D. Ciullo, and M. Meo. Optimal energy savings in cellular access networks. IEEE GreenComm, 2009.

[2] B.D. Higgins, A. Reda, T. Alperovich, J. Flinn, T.J. Giuli, B. Noble, and D. Watson. Intentional Networking: Opportunistic Exploitation of Mobile Network Diversity. ACM MobiCom, pages 73-84, 2010.

[3] A. Balasubramanian, R. Mahajan, and A. Venkataramani. Augmenting Mobile 3G using WiFi. ACM MobiSys, pages 209-222, 2010.

[4] M. Buddhikot, G. Chandranmenon, S. Han, Y.W. Lee, S. Miller, and L. Salgarelli. Integration of 802.11 and Third-Generation Wireless Data Networks. IEEE INFOCOM, pages 503-512, 2003.

[5] P. Antoniadis, B. Le Grand, A. Satsiou, L. Tassiulas, R.L. Aguiar, J.P. Barraca, and S. Sargento. Community Building over Neighborhood Wireless Mesh Networks. IEEE Technology and Society, 27(1), 2008.

[6] X. Zhou, S. Gandhi, S. Suri, and H. Zheng. eBay in the Sky: Strategyproof Wireless Spectrum Auctions. ACM MobiCom, pages 2-13, 2008.

[7] X. Zhou and H. Zheng. TRUST: A General Framework for Truthful Double Spectrum Auctions. IEEE INFOCOM, pages 999-1007, 2009.

[8] G.S. Kasbekar and S. Sarkar. Spectrum Auction Framework for Access Allocation in Cognitive Radio Networks. ACM MobiHoc, pages 18411854, 2009.

[9] S. Sengupta and M. Chatterjee. An Economic Framework for Dynamic Spectrum Access and Service Pricing. IEEE/ACM Trans. on Networking, 17(4):1200-1213, 2009

[10] J. Jia, Q. Zhang, Q. Zhang, and M. Liu. Revenue Generation for Truthful Spectrum Auction in Dynamic Spectrum Access. ACM MobiCom, pages 3-12, 2009.

[11] S. Eidenbenz, G. Resta, and P. Santi. The COMMIT Protocol for Truthful and Cost-efficient Routing in Ad hoc Networks with Selfish Nodes. IEEE Trans. on Mobile Computing, 7(1):19-33, 2008.

[12] J. Jaramillo and R. Srikant. DARWIN: Distributed and Adaptive Reputation Mechanism for Wireless Ad hoc Networks. ACM MobiCom, pages 87-98, 2007.

[13] W. Wang and X.Y. Li. Low-Cost Routing in Selfish and Rational Wireless Ad hoc Networks. IEEE Trans. on Mobile Computing, 5(5):596-607, 2006.

[14] Y. Wu, S. Tang, P. Xu, and X.Y. Li. Dealing With Selfishness and Moral Hazard in Non-Cooperative Wireless Networks. IEEE Trans. on Mobile Computing, 9(3):420-434, 2009.

[15] S. Zhong and F. Wu. On Designing Collusion-Resistant Routing Schemes for Non-Cooperative Wireless Ad hoc Networks. ACM MobiCom, pages 278-289, 2007.

[16] R. Jain and J. Walrand. An Efficient Mechanism for Network Bandwidth Auction. IEEE NOMS, pages 227-234, 2008.

[17] F. Fu and U.C. Kozat. Wireless Network Virtualization as a Sequential Auction Game. IEEE INFOCOM, pages 1945-1953, 2010.

[18] D. Lehmann, L.I. Oćallaghan, and Y. Shoham. Truth Revelation in Approximately Efficient Combinatorial Auctions. Journal of the ACM (JACM), 49(5):577-602, 2002. 